\title{
Effect of visibility of the loci on recall using the method of loci
}

\author{
SIMON KEMP and CHRISTOPHER D. van der KROGT \\ University of Canterbury, Christchurch, New Zealand
}

(K. T. Strongman, sponsor)

Two experiments, in which the visibility of the loci was varied, investigated the method of loci. Results of the experiments showed recall to be unaffected by whether the loci were visible (i.e., physically present) during both learning and recall phases or invisible (and hence imagined) during both phases. Recall was adversely affected, relative to these groups, if the loci were visible during learning but not during recall.

Mnemonic devices have been known and used at least since classical times (Yates, 1966). One of the oldest and best known of these devices is the method of loci, in which the subject first learns to visualize an ordered series of places (loci). Each object or noun to be remembered is then visualized in association (often bizarre) with the corresponding locus. To recall, the subject mentally visualizes the loci in order and, in so doing, retrieves the associated image.

The method is undoubtedly effective for remembering lists of unrelated objects or nouns (e.g., Bellezza, 1981; Ross \& Lawrence, 1968). Recent research has also investigated the question of how the method works and has identified some necessary and desirable factors for its effective use. Thus, for example, it appears that the associating images need not be bizarre (Bower, 1970; Higbee, 1979); and the subject does not necessarily have to memorize and be familiar with the loci. Instead, he or she can use a memory map of fictitious locations supplied by the experimenter (Briggs, Hawkins, \& Crovitz, 1970; Crovitz, 1969). It now appears that the ordering of the images is a definite factor in the method's effectiveness: Formation of images alone does not provide such a high rate of recall as is achieved by the method of loci or by the similar peg system (Bower \& Reitman, 1972; Roediger, 1980).

One important factor is whether or not the loci are physically present and visible. This factor is important not only in itself, but also because it relates to variables that have been investigated previously. If the loci are visible, then their ordering, which affects performance using the method, is less likely to be forgotten or confused than if the loci themselves must be remembered. It is noteworthy, in this context, that the "Gorky Street" procedure of

The project was supported by the New Zealand Department of Labour under the PEP scheme. The authors are grateful for useful discussion with Paul Russell and Ken Strongman. The authors' mailing address is: Department of Psychology, University of Canterbury, Christchurch, New Zealand.
Briggs et al. (1970) and Crovitz (1969) provides ordering information and verbal cues throughout learning and recall-subjects must memorize only their images. The relevance of the issue is underlined by an experiment undertaken by Bellezza and Reddy (1978). Subjects were instructed to form images of words together with either familiar or unfamiliar loci. Their recall performance was measured both with and without loci cues (subjects' own or supplied lists of loci). Cuing produced little effect when familiar loci were used but considerable improvement, to the level obtained with the familiar loci, when unfamiliar loci were used.

In the research described below, experimental subjects were instructed to use loci from the same environment. Visibility of the loci during learning and recall was varied between experimental groups.

\section{EXPERIMENT 1}

\section{Method}

Subjects. The subjects were 77 students in the 1st-year psychology course. They were recruited from four different laboratory groups, each providing subjects for one experimental condition.

Materials. Three lists (Lists 1, 2, and 3) of 25 words each were compiled from words with imagery and concreteness values greater than 6.0 and with frequencies of occurrence between 10 and 50 per million as reported by Paivio, Yuile, and Madigan (1968). The same lists were used for each experimental group. Auditory presentation of the words was used. Words spoken by the first author were recorded at the rate of one word every $8 \mathrm{sec}$. A large laboratory, familiar to the subjects, with a varied collection of cupboards, posters, etc., was used to provide the loci. An additional room, whose design, furnishing, and decoration were different from those of the laboratory, was also available when the subjects were denied access to visible loci.

Procedure. Four groups were designated as follows: Control (Group C)-No instruction in the method of loci was given. They remained in the laboratory throughout. Group E1-The subjects had visible loci (i.e., were in the laboratory) during learning and recall of the word lists. Group E2-The subjects had visible loci during learning but not during recall. Group E3-The subjects had visible loci during neither learning nor recall (that is, they learned and recalled the word lists in the other room).

All groups participated in two experimental sessions separated by an interval of 2 weeks. The first session was a pretest, in which the sub- 
jects were instructed to remember List 1 . Three minutes after hearing the word list, they were instructed to write down on a numbered sheet the words that they had heard. Three minutes were allotted for the recall.

Group $\mathrm{C}$ was given identical instructions in the second experimental session when they were required to learn Lists 1 and 2 . Timing for word presentation and recall was as in the pretest; there was a further 3-min interval between the end of the recall period for List 2 and the presentation of List 3.

At the beginning of the second experimental session, for Groups E1 and E2, or 1 week after the pretest, for Group E3, the subjects were instructed in the use of the method of loci. They were required to identify 25 separate visible loci, preferably ordered in a head turn from the left-hand to the right-hand back corner of the room. They were required to list the loci chosen on paper. Fifteen minutes were allowed for the subjects to learn the loci.

After the 15-min period, Group E1 was tested with Lists 2 and 3. The subjects, who were instructed to use the visible loci, were permitted to use their lists of loci to remember their order, and remained in their places throughout. Group E2 was told that, after presentation of Lists 2 and 3, recall would take place elsewhere. They were permitted to use their lists of loci during presentation but not during recall.

Group E3 was instructed to take their lists of loci home with them and to try to memorize them during the next week, after which they were to be tested in a different room. They were requested to return, if possible, to the lab for revision of the loci. At the beginning of the experimental session 1 week later, they were given $10 \mathrm{~min}$ to revise their loci, following which testing and recall of Lists 2 and 3 took place in the additional room. The subjects were not permitted to use their loci lists.

Timing of presentation and recall was the same for all groups.

\section{Results and Discussion}

Table 1 presents the important results of the experiment. The dependent variable is the average number of words correctly recalled. Analyses of variance showed no significant effect of groups on the pretest $[\mathrm{F}(3,73)=0.31$, $\mathrm{p}>.05]$. Analyses of variance on the results of the main tests showed significant effects of groups $[\mathrm{F}(3,73)=5.13$, $\mathrm{p}<.05]$ and lists $[\mathrm{F}(1,73)=27.44, \mathrm{p}<.05]$, but not the lists $\times$ groups interaction $[F(3,73)=0.10, p>.05]$.

The most important results concern the effect of different experimental conditions. A planned comparison between the experimental groups (Groups E1, E2, and E3) and Group $C$ showed a significant effect $[F(1,73)=$ $12.21, \mathrm{p}<.05$ ]; instruction in the method of loci produced an improvement in the number of words correctly recalled.

A multiple range test (Tukey HSD, $p=.05$ ) showed no significant differences among Groups E1, E2, and E3. The results shown in Table 1, however, suggest that such

Table 1

Results of Experiment 1

\begin{tabular}{cccrcc}
\hline Group & $\mathrm{n}$ & $\begin{array}{c}\text { List 1 } \\
\text { (Pretest) }\end{array}$ & \multicolumn{1}{c}{ List 2 } & List 3 & $\begin{array}{c}\text { Average } \\
\text { of Lists 2 and 3 }\end{array}$ \\
\hline C & 14 & 11.2 & 9.4 & 11.9 & 10.6 \\
E1 & 21 & 10.7 & 14.6 & 16.5 & 15.6 \\
E2 & 26 & 11.7 & 12.6 & 14.8 & 13.7 \\
E3 & 16 & 11.9 & 15.3 & 17.4 & 16.4 \\
\hline
\end{tabular}

Note-The table shows the average number of words correctly remembered for each list and experimental group. The average number recalled on the test lists (Lists 2 and 3) is also given. differences might exist. In particular, it seems likely that visible loci during learning and recall do not produce a greater improvement in recall than that obtained with wholly imagined loci. Moreover, having loci visible during learning but not during recall may actually produce worse recall than not having them visible at any stage. Such conclusions, however, are only suggestive and, in addition, are confounded by the fact that Group E2, in this experiment, must change rooms, and thus be more liable to distraction than the other groups.

Experiment 2 was carried out to test the suggestive results of Experiment 1.

\section{EXPERIMENT 2}

\section{Method}

Subjects. The subjects were 164 students in the 1st-year psychology course, recruited from eight different laboratory groups, two groups being assigned to each experimental group. Assignment was performed on the basis of the pretest and was designed to keep average pretest scores as similar as possible across conditions. The subjects were distinct from those in Experiment 1.

Materials. Lists 1, 2, and 3 were constructed according to the same criteria that had been used in Experiment 1 . They were recorded as spoken by the second author at the rate of one every $10 \mathrm{sec}$. The same rooms that had been used in Experiment 1 were used here.

Procedure. As in Experiment 1, four experimental groups were designated. All groups participated in three experimental sessions with intervals of 2 weeks and 1 week between them. The first session was a pretest in which List 1 was presented with instructions and timing similar to those in Experiment 1.

At the beginning of the second session, Groups E1, E2, and E3 were instructed in the use of the method of loci and were required to construct lists of the loci as before. The subjects in all experimental groups could use their loci lists while the words were presented. The lists were, however, in no case available for the recall. List 2 was presented to the four experimental groups and recall was assessed according to the same timing and room designations as in Experiment 1. One new feature was that, in the interval between presentation and recall, Groups $\mathrm{C}, \mathrm{E} 1$, and E3 were moved out of the room that they occupied and back again, in order to provide a distraction comparable to that suffered by Group E2.

List 3 was presented in the final session. The experimental groups were handed back their lists of loci and were asked to revise them for $10 \mathrm{~min}$. Presentation and recall for these groups, as well as for Group C, then proceeded as in the preceding session.

As suggested by Perensky and Senter (1974), the subjects were asked how they remembered words in the pretest; their reports were scored for the use or otherwise of a mnemonic technique. The subjects were also asked whether they had used the method of loci (or, for Group C, any mnemonic technique) for List 3.

\section{Results and Discussion}

The main results are presented in Table 2. Analyses of variance on the results of Lists 2 and 3 showed significant effects of experimental conditions $[\mathrm{F}(3,160)=3.73$, $\mathrm{p}<.05]$ but not lists $[\mathrm{F}(1,160)=0.81, \mathrm{p}>.05]$. There was a significant lists $\times$ conditions interaction $[F(3,160)$ $=4.22, \mathrm{p}<.05]$. Planned comparisons were carried out as suggested by the results of Experiment 1. Instruction in the method of loci had a significant effect [Groups E1, E2, and E3 vs. Group C: $F(1,160)=4.22, p<.05]$. Having the loci visible during learning but not during 
Table 2

Results of Experiment 2

\begin{tabular}{cccccc}
\hline Group & $\mathrm{n}$ & $\begin{array}{c}\text { List 1 } \\
\text { (Pretest) }\end{array}$ & List 2 & List 3 & $\begin{array}{c}\text { Average } \\
\text { of Lists 2 and 3 }\end{array}$ \\
\hline C & 41 & 13.9 & 17.2 & 16.2 & 16.7 \\
E1 & 41 & 14.1 & 19.5 & 19.6 & 19.5 \\
E2 & 47 & 13.9 & 16.8 & 17.2 & 17.0 \\
E3 & 35 & 14.6 & 17.6 & 19.1 & 18.3 \\
\hline
\end{tabular}

Note-The table shows the average number of words correctly recalled for each experimental group and list. The average score on Lists 2 and 3 is also given.

recall produced a significantly worse performance than when the loci were either continually or never visible [Group E2 vs. Groups E1 and E3: F $(1,160)=5.59$, p $<.05]$. Whether the loci were always or never visible had no significant effect [Group E1 vs. Group E3: $\mathrm{F}(1,160)=1.39$, n.s.], and there was no significant difference between Group E2 and Group $C[F(1,160)=$ 0.13 , n.s.]. In general, these results agree with those expected from Experiment 1.

Comparison of the pretest results from Experiments 1 and 2 (cf. Tables 1 and 2) reveals the odd result that the subjects in Experiment 2 generally started off recalling better than those in Experiment 1. Such a difference may have arisen from procedural differences between the experiments. A likely cause of some of the difference, however, was a change in the undergraduate teaching program: The subjects in Experiment 2 had been introduced briefly to mnemonic techniques. Of the 160 subjects who answered the question, 39 reported having used a mnemonic technique. Their average score was 17.3 words correctly remembered, whereas that for the nonusers was $13.2[\mathrm{~F}(1,158)=45.6, \mathrm{p}<.05]$. Table 2 shows that not only the experimental groups, but also the control group, performed better on Lists 2 and 3 than on the pretest. This seems to have been due partly to adoption of mnemonic techniques by the control group: 9 subjects used a mnemonic technique for List 1 , and 14 did so for List 3 . Again, the subjects who used the technique performed better than those who did not: Average scores were 17.0 and 13.4, respectively, on List 1 and 20.8 and 13.5 on List 3.

Most subjects who had been instructed in the method of loci reported following it for List 3. Of the 13 who did not, 7 were in Group E2 and 4 in Group E3, and their average score was 13.7 words. Thus, the relatively poor performance of Group E2 may have been, in part, the consequence of difficulty in using the method in this variation.

The significant interaction of tests and conditions found in Experiment 2 seems, from an examination of Table 2, to have resulted largely from an improvement in the performance of Group E3 over the two tests.

\section{DISCUSSION}

The similarity of the results reported here provides extra suppport for the general conclusions that may be derived from them. These conclusions are:

First, whether the loci are visible throughout learning and recall or whether they are imagined in both phases does not affect the effectiveness of recall.

Second, the effectiveness of the method of loci is impaired if the loci are visible during learning but imagined during recall.

The first of these results is subject to the qualification that the loci are memorized. The interaction found in Experiment 2 between the effects of lists and conditions suggests that loci had not been adequately memorized prior to presentation of List 2. Words in List 3, in Experiment 2, were, however, almost equally well recalled by Groups E1 and E3. In Experiment 1, in which more time was initially allotted Group E3 for memorizing the loci, this interaction did not occur.

The second general result suggests that imagined and visible loci are not interchangeable. The simplest explanation of the result is that images of the loci, although themselves stable, differ in some way from the visible loci, and, in effect, form a different array of loci. Attempting to recall the associated words by stepping through the imagined array would be less effective because the words would not be associated with that array, but with the related (visible) one.

One last issue should be mentioned. The results reported here have a practical implication. They suggest that memorized loci should generally be used in preference to visible loci. Even if the latter are available for recall, performance appears no better. If, for some reason, they become unavailable for recall, a deterioration in performance relative to that expected with memorized loci is likely to result.

\section{REFERENCES}

BellezzA, F. S. (1981). Mnemonic devices: Classification, characteristics, and criteria. Review of Educational Research, 51, 247-275.

BellezZA, F. S., \& ReDdY, B. G. (1978). Mnemonic devices and natural memory. Bulletin of the Psychonomic Society, 11, 277-280.

Bower, G. H. (1970). Analysis of a mnemonic device. American Scientist, 58, 496-510.

Bower, G. H., \& ReITMAN, J. S. (1972). Mnemonic elaboration in multilist learning. Journal of Verbal Learning and Verbal Behavior, 11, 478-485.

Briggs, G. G., Hawkins, S., \& Crovitz, H. F. (1970). Bizarre images in artificial memory. Psychonomic Science, 19, 353-354.

Crovitz, H. F. (1969). Memory loci in artificial memory. Psychonomic Science, 16, 82-83.

HigbeE, K. L. (1979). Recent research on visual mnemonics: Historical roots and educational fruits. Review of Educational Research, 49, 611-629.

Paivio, A., Yuille, J. C., \& Madigan, S. A. (1968). Concreteness, imagery, and meaningfulness values for 925 nouns. Journal of Experimental Psychology Monographs, 76(1, Pt. 2).

Perensky, J. J., \& Senter, R. J. (1970). The effect of subjects conforming to mnemonic instructions. Journal of Psychology, 74, 15-20.

RoEDiger, H. L. (1980). The effectiveness of four mnemonics in ordering recall. Journal of Experimental Psychology: Human Learning and Memory, 6, 558-567.

Ross, J., \& LAWRENCE, K. A. (1968). Some observations on memory artifice. Psychonomic Science, 13, 107-108.

YATES, F. A. (1966). The art of memory. London: Routledge \& Kegan Paul.

(Manuscript received for publication December 28, 1984.) 